\title{
Optimal Ratio of Wnt3a Expression in Human Mesenchy- mal Stem Cells Promotes Axonal Regeneration in Spinal Cord Injured Rat Model
}

\author{
Hyung Ho Yoon, ${ }^{1, *}$ Hyang Ju Lee, ${ }^{2, *}$ Joongkee Min, ${ }^{1, *}$ Jeong Hoon Kim, ${ }^{1, *}$ Jin Hoon Park, Ji Hyun Kim, ${ }^{3,4}$ Seong Who Kim, ${ }^{2}$ \\ Heuiran Lee, ${ }^{3,4}$ Sang Ryong Jeon ${ }^{1}$ \\ Department of Neurological Surgery, Asan Medical Center, University of Ulsan College of Medicine, Seoul, Korea \\ Department of Biochemistry and Molecular Biology, ${ }^{2}$ University of Ulsan College of Medicine, Seoul, Korea \\ Department of Microbiology, University of Ulsan College of Medicine, Seoul, Korea \\ Bio-Medical Institute of Technology, ${ }^{4}$ University of Ulsan College of Medicine, Seoul, Korea
}

Objective : Through our previous clinical trials, the demonstrated therapeutic effects of MSC in chronic spinal cord injury (SCI) were found to be not sufficient. Therefore, the need to develop stem cell agent with enhanced efficacy is increased. We transplanted enhanced Wnt3asecreting human mesenchymal stem cells (hMSC) into injured spines at 6 weeks after $\mathrm{SCl}$ to improve axonal regeneration in a rat model of chronic SCI. We hypothesized that enhanced Wnt3a protein expression could augment neuro-regeneration after SCl.

Methods : Thirty-six Sprague-Dawley rats were injured using an Infinite Horizon (IH) impactor at the T9-10 vertebrae and separated into five groups : 1) phosphate-buffered saline injection (injury only group, $n=7$ ); 2) hMSC transplantation (MSC, $n=7$ ); 3) hMSC transfected with pLenti vector (without Wnt3a gene) transplantation (pLenti-MSC, n=7); 4) hMSC transfected with Wnt3a gene transplantation (Wnt3a-MSC, n=7); and 5) hMSC transfected with enhanced Wnt3a gene (1.7 fold Wnt3a mRNA expression) transplantation (1.7 Wnt3a-MSC, $\mathrm{n}=8$ ). Six weeks after $\mathrm{SCl}$, each $5 \times 10^{5}$ cells $/ 15 \mu \mathrm{L}$ at 2 points were injected using stereotactic and microsyringe pump. To evaluate functional recovery from $\mathrm{SCl}$, rats underwent Basso-Beattie-Bresnahan (BBB) locomotor test on the first, second, and third days post-injury and then weekly for 14 weeks. Axonal regeneration was assessed using growth-associated protein 43 (GAP43), microtubule-associated protein 2 (MAP2), and neurofilament (NF) immunostaining.

Results : Fourteen weeks after injury (8 weeks after transplantation), BBB score of the 1.7 Wnt3a-MSC group (15.0 \pm 0.28 ) was significantly higher than that of the injury only (10.0 \pm 0.48$)$, MSC (12.57 \pm 0.48$)$, pLenti-MSC (12.42 \pm 0.48$)$, and Wnt3a-MSC (13.71 \pm 0.61$)$ groups $(p<0.05)$. Immunostaining revealed increased expression of axonal regeneration markers GAP43, MAP2, and NF in the Wnt3a-MSC and 1.7 Wnt3a-MSC groups.

Conclusion : Our results showed that enhanced gene expression of Wnt3a in hMSC can potentiate axonal regeneration and improve functional recovery in a rat model of chronic SCI.

Key Words : Spinal cord regeneration · Recovery of function · Mesenchymal stem cells · Spinal cord injuries · Wnt3a.

- Received : January 4, 2021 •Revised : March 31, 2021 •Accepted : April 7, 2021

- Address for reprints : Sang Ryong Jeon

Department of Neurological Surgery, Asan Medical Center, University of Ulsan College of Medicine, 88 Olympic-ro 43-gil, Songpa-gu, Seoul 05505, Korea

Tel : +82-2-3010-3550, Fax : +82-2-476-6738, E-mail : srjeon@amc.seoul.kr, ORCID : https://orcid.org/0000-0002-8340-7978

\section{Heuiran Lee}

Department of Microbiology, University of Ulsan College of Medicine, 88 Olympic-ro 43-gil, Songpa-gu, Seoul 05505, Korea

Tel : +82-2-3010-4286, E-mail : heuiran@gmail.com, ORCID : https://orcid.org/0000-0002-4241-5664

${ }^{*}$ These authors contributed equally to this work.

This is an Open Access article distributed under the terms of the Creative Commons Attribution Non-Commercial License (http://creativecommons.org/licenses/by-nc/4.0) which permits unrestricted non-commercial use, distribution, and reproduction in any medium, provided the original work is properly cited. 


\section{INTRODUCTION}

Spinal cord injury (SCI) results in neurological deficits in both sensory and motor functions, leading to inevitable disabilities $^{(4)}$. Currently, there is no available treatment that can revert to an intact state. In a previous study, we focused on axonal regeneration for repair of SCI, and evaluated the efficacy of Wnt3a-secreting cell transplants in regenerating injured axons; we observed increased axonal regeneration and recovery of motor function in a sub-acute $\mathrm{SCI}^{21,25,26)}$. Wnt proteins are a family of signaling molecules that play roles in development, cell differentiation, and proliferation ${ }^{8,23}$. They are also crucial in multifunctional axon guidance ${ }^{8,28}$. Wnt signaling through glycogen synthase kinase-3 is involved in axon remodeling ${ }^{8,28)}$. Upregulation of Wnt signaling activates Ryk, a Wnt receptor in corticospinal neurons, resulting in axon retraction after injury ${ }^{18}$. Wntl and Wnt3a modulate spinal cord dorsal interneuron differentiation, and Wnt3a has been implicated in neurogenesis ${ }^{7)}$.

Currently, high proportion of candidates for cell therapy in SCI is chronic status, including those in our previous clinical trials ${ }^{16,20)}$. However, there is no gold standard for determining the chronic phase period in a rat model of SCI. To standardize a practical approach to chronic SCI, we need to identify the most suitable chronic phase in a rat model of SCI. In our pilot study, we injected mesenchymal stem cells (MSC) at 4 weeks after SCI, but found that chronic SCI manifests 6 weeks after SCI; behavioral improvement plateaued at 6 weeks after SCI in most injury only group (data not shown). Therefore, in this study, we transplanted Wnt3a-secreting human mesenchymal stem cells (hMSC) into a contusion site 6 weeks after SCI. We also hypothesized that enhanced Wnt3a protein expression could induce more pronounced neuro-regenerative effects even in the chronic condition. Thus, this study aimed to identify the ideal expression ratio of the Wnt3a gene transfected into MSC to maximize neuro-regenerative effects in a chronic rat SCI model.

\section{MATERIALS AND METHODS}

All procedures complied with the guidelines of the Institutional Animal Care and Use Committee of the Asan Institute for Life Sciences and were approved by the Ethics Committee for Animal Experiments of the Asan Institute for Life Sciences (Seoul, Korea).

\section{Cloning and lentivirus production}

We extracted human Wnt3a cDNA from a pcDNA/Wnt3a plasmid (\#35908; Addgene, Cambridge, MA, USA) using restriction enzymes. We inserted extracted DNA into a pLentisuCMV-Rsv-GFP plasmid (GenTarget Inc., San Diego, CA, USA) to create a pLenti-suCMV-Rsv-GFP/human Wnt3a plasmid. For lentivirus production, we used a Neon transfection System (Thermo Fisher Scientific, Waltham, MA, USA), mixing pLenti-suCMV-Rsv-GFP/human Wnt3a, pMD2G (\#12259; Addgene), and psAX2 (\#12260; Addgene) in Lenti-X 293T (Takara Bio, Shiga, Japan) cells. After 2 days of incubation, we harvested the supernatant containing the concentrated virus using the WelProt Virus Concentration Reagent (WelGENE, Daegu, Korea). Lentivirus titers were measured using the Lenti-X qRT-PCR Titration kit (Takara Bio).

\section{Genetically modified MSC}

MSCs derived from human umbilical cord blood (KS-R218-3G1-N; Kangstem Biotech, Seoul, Korea) were multiplied and cultivated in a KSB-3 Complete Medium kit (Kangstem Biotech) at $37^{\circ} \mathrm{C}$ and $5 \% \mathrm{CO}_{2}$. These cells were infected with the prepared lentivirus to insert the Wnt3a gene. After cultivation in media mixed with $2.5 \mu \mathrm{L} / \mathrm{mL}$ puromycin for an additional 48 hours, the infected cells were identified by GFP expression through confocal microscopy (Carl Zeiss Meditec, Jena, Germany). Wnt3a gene copy number in the MSCs was measured using the Lenti-X Provirus Quantitation kit (Takara Bio).

\section{Experimental animals}

Fifty female Sprague-Dawley rats (Orient Bio Inc., Seongnam, Korea), weighing 230-250 $\mathrm{g}$ at the beginning of the experiment, were housed in a room with a $12 \mathrm{~h} / 12 \mathrm{~h}$ light/dark cycle and free access to food and water.

\section{$\mathrm{SCl}$ and experimental groups}

Surgical procedures were performed under general anesthesia induced by $1.5 \%$ isoflurane (Piramal Critical Care, Inc., Bethlehem, PA, USA). After skin incision, the vertebral column was exposed between T9 and T10, and a laminectomy was performed at the $\mathrm{T} 9$ level. The rats received a moderate SCI induced by an Infinite Horizon (IH) impactor (250-kKdyn; 
Precision System Instrumentation, Fairfax, VA, USA $)^{27}$. After SCI, the contusion site was washed with normal saline, and the skin was sutured. Cefazolin $(10 \mathrm{mg} / \mathrm{kg})$ and ketoprofen $(5 \mathrm{mg} / \mathrm{kg}$ ) were subcutaneously administered for antibiotic prophylaxis once a day for 3 days, and each rat was housed individually. The rat's bladders were emptied manually thrice daily until spontaneous voiding was recovered. At the beginning of the experiment, there were 50 animals, but 14 animals were excluded due to death or injury failure. Rats were blindly separated into five groups : 1) phosphate-buffered saline (PBS) injection only group (injury only group, $\mathrm{n}=7$ ); 2) hMSC transplantation group (MSC group, $\mathrm{n}=7$ ); 3) hMSC transfected with pLenti vector (without Wnt3a gene) transplantation group (pLenti-MSC group, $\mathrm{n}=7$ ); 4) hMSC transfected with Wnt3a gene transplantation group (Wnt3a-MSC group, $n=7$ ); and 5) hMSC transfected with enhanced Wnt3a gene transplantation group (1.7 Wnt3a-MSC group, $\mathrm{n}=8$ ).

\section{Cell transplantation}

Six weeks after SCI, all rats were anesthetized with $2 \%$ isoflurane. After spinal cord re-exposure, hMSCs were delivered at a rate of $1 \mu \mathrm{L} / \mathrm{min}$ using a $31 \mathrm{G}$ Hamilton microsyringe and an automated microsyringe pump (Harvard Apparatus, Holliston, MA, USA), which are fixed to the stereotactic device. The cells were inserted into the center of the contusion site at a depth of $1 \mathrm{~mm}$ from the dorsal dura. The number of injected cells in each group for transplantation was $5 \times 10^{5}$ cells $/ 15 \mu \mathrm{L} /$ point at 2 points (the injury epicenter and $2 \mathrm{~mm}$ proximal to the epicenter). Total cell numbers were $1 \times 10^{6}$ cells/30 $\mu \mathrm{L}$. After injection, the needle was kept in place for 5 minutes to prevent the solution from flowing backward and was then retracted over a subsequent 5 minutes.

\section{Behavioral test}

After preparing the rat SCI models, we assessed the neurological function of each rat using the Basso-Beattie-Bresnahan (BBB) locomotor rating scale ${ }^{1)}$. Behavioral tests were performed on the first, second, and third day after injury, and then weekly for 14 weeks. Before the BBB test, each rat was allowed to adapt to the open field $(90 \times 90 \mathrm{~cm}, 10 \mathrm{~cm}$ wall height). Once the rat demonstrated acclimatization by walking continuously in the open field for 10 minutes, an examiner blinded to group-identifying information assessed the locomotor performance of each rat using the BBB scale. The be- havioral test scores of all groups were analyzed by one-way analysis of variance (ANOVA), followed by a post hoc TukeyKramer multiple comparison test. All statistical analyses were performed using Statistical Package for Social Sciences (SPSS) ver. 12.0.1 (SPSS Inc., Chicago, IL, USA). Statistical significance was set at $p<0.05$.

\section{Real-time quantitative polymerase chain reaction}

Total RNA was isolated from MSCs using TRIzol Reagent (Thermo Fisher Scientific) in accordance with the manufacturer's protocol. Total RNA aliquots $(2 \mu \mathrm{g})$ were then reverse transcribed using a TOPscript cDNA Synthesis Kit (Enzynomics, Daejeon, Korea). Real-time quantitative polymerase chain reaction (qPCR) was performed on a CFX Connect Real-Time PCR Detection System (Bio-rad, Hercules, CA, USA). Gene-specific primer sets were designed, and the following primers were used to amplify the target mRNA : Wnt3a (F) 5'-CATGAACCGCCACAACAAC-3' and (R) 5'-TCGCAGAAGTTGGGCGA'-3', $\beta$-actin (F) 5'-CTTCGGGAAGGAGCTCAAA-3' and (R) 5'-TGCCTCGTTGTTGTGCAAG-3'. Gene expression was normalized to that of $\beta$-actin, which was used as an internal control. Relative quantities of the mRNA of interest were calculated using the comparative threshold cycles $\left(2^{-\Delta \Delta C t}\right)$ method.

\section{Western blotting}

Cells were lysed in radioimmunoprecipitation assay (RIPA) lysis buffer (Thermo Fisher Scientific) containing the Halt protease inhibitor cocktail (Thermo Fisher Scientific). Equal amounts of protein $(10 \mu \mathrm{g})$ were resolved using sodium dodecyl sulfate-polyacrylamide gel electrophoresis (SDS-PAGE) and transferred onto a nitrocellulose membrane. Each membrane was blocked in $0.1 \%$ Tris-buffered saline with Tween-20 (TBST) containing 5\% bovine serum albumin (BSA) and incubated with indicated primary antibodies against Wnt3a (Novus Biologicals, Centennial, CO, USA), $\beta$-catenin (Cell Signaling Technology, Danvers, MA, USA), and $\beta$-actin (Sigma-Aldrich, St. Louis, MO, USA). The blots were developed using Advansta Western Bright ECL HRP Substrate Kits (Advansta, San Jose, CA, USA) and detected using a C-DiGit Blot Scanner (LI-COR, Lincoln, NE, USA).

\section{Immunofluorescence staining}

For immunofluorescence analysis, all rats were sacrificed 
and perfused with PBS and 4\% paraformaldehyde (PFA; Sigma-Aldrich) 7 weeks after transplantation. The lesion sites of the spinal cords were extracted and fixed with 4\% PFA for 6 hours, followed by incubation with 30\% sucrose in PBS overnight. Serial longitudinal spinal cord sections were collected (10 $\mu \mathrm{m}$ thickness) utilizing a Cryotome (Microm, Waldorf, Germany) and mounted on poly-L-lysine-coated Superfrost Plus slides (Matsunami, Osaka, Japan). The sections were washed three times with Tris-buffered saline (TBS), then blocked with TBS containing 5\% BSA and 0.1\% Triton X-100 for 1 hour at room temperature. The sections were incubated overnight with primary antibody at $4^{\circ} \mathrm{C}$ and washed three times with TBS, then incubated with fluorescent-conjugated secondary antibody (1 : 1000; Invitrogen, Carlsbad, CA, USA). The primary antibodies used were : neurofilament (NF, 1 : 200; Abcam, Cambridge, UK), growth-associated protein 43 (GAP43, 1 : 200; Abcam), and microtubule-associated protein 2 (MAP2, $1: 200$; Abcam). Fluorescent images were obtained using a confocal microscope (Carl Zeiss, Oberkochen, Germany) with ZEN microscope software (Carl Zeiss). Fluorescent intensity was analyzed using ImageJ program (National Institutes of Health and the Laboratory for Optical and Computational Instrumentation (Madison, University of Wisconsin).

\section{Cytokine analysis}

After 48 hours incubation in opti-MEM medium (Gibco; Thermo Fisher Scientific), supernatants of MSC, hMSC transfected with Wnt3a gene, hMSC transfected with enhanced Wnt3a gene were collected. Supernatants were biotin-labelled and hybridized onto human L1000 glass slide arrays (RayBiotech, Peachtree Corners, GA, USA). Detected signals were then normalized using BCA protein assay kit.

\section{RESULTS}

\section{Development of enhanced Wnt3a-MSC}

In a previous study, we manufactured Wnt3a-expressing MSC (baseline gene expression per cell), but these cells could not statistically improve behavioral results when compared to pure MSC (data not shown). Thus, we needed an improved Wnt3a-MSC that could secret more Wnt3a protein, allowing for more effective signaling for axon regeneration. We developed a new Wnt3a-MSC by increasing the quantity of Wnt3a gene for transfecting into MSC ( 2 fold and 4 fold). The results shown in Fig. 1 indicate that 2 fold Wnt3a gene transfected MSC (2× Wnt3a-MSC) showed 1.7 fold Wnt3a mRNA expression level as compared to baseline Wnt3a gene transfected MSC (1× Wnt3a-MSC), while 4 fold Wnt3a gene transfected MSC (4× Wnt3a-MSC) showed 2.4 fold Wnt3a mRNA expression level as compared to $1 \times$ Wnt3a-MSC. Interestingly, we found that $2 \times$ Wnt3a-MSC secreted more Wnt3a proteins even compared to the $4 \times$ Wnt3a-MSC (Fig. 2); the $2 \times$ Wnt3aMSC showed 3.25 fold Wnt3a protein level as compared to $1 \times$ Wnt3a-MSC (Fig. 2). The $2 \times$ Wnt3a-MSC also showed a 2.2 fold increase in $\beta$-catenin expression level when compared to $1 \times$ Wnt3a-MSC (Fig. 3). Given these observations, we selected $2 \times$ Wnt3a-MSC to be the new enhanced Wnt3a-MSC, henceforth referred to as $1.7 \mathrm{Wnt} 3 \mathrm{a}-\mathrm{MSC}$. In the $1 \times \mathrm{Wnt} 3 \mathrm{a}-\mathrm{MSC}$, $3.5 \times 10^{4}$ copies of the virus were utilized per MSC, whereas in the 1.7 Wnt3a-MSC, $7 \times 10^{4}$ copies of virus were utilized per MSC. After each round of transfection, we quantified the provirus copy number using the Lenti-X Provirus Quantitation kit. We detected 13.02 provirus copies/cell in $1 \times$ Wnt3a-MSC, and 22.19 provirus copies/cell in 1.7 Wnt3a-MSC.

\section{Enhanced Wnt3a gene expression in hMSC pro- motes behavioral recovery}

We injected PBS (injury only group), hMSC (MSC group), hMSC transfected with pLenti vector (pLenti-MSC group), hMSC transfected with Wnt3a genes (Wnt3a-MSC group),

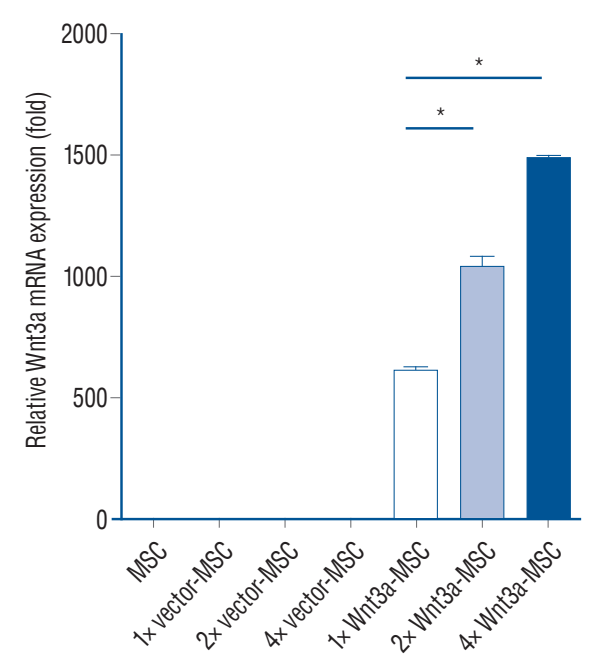

Fig. 1. Wnt3a mRNA expression in each transfection condition. $2 \times$ Wnt3a-MSC showed 1.7 fold Wnt3a mRNA expression level, and $4 \times$ Wnt3a-MSC showed 2.4 fold Wnt3a mRNA expression level as compared to $1 \times$ Wnt3a-MSC. ${ }^{*} p<0.001$. MSC : mesenchymal stem cells. 
and hMSC transfected with enhanced Wnt3a genes (1.7 Wnt3a-MSC group) in the respective groups 6 weeks after SCI. We found that the 1.7 Wnt3a-MSC group showed significant behavioral recovery compared with all other groups $(p<0.05)$. BBB score of the 1.7 Wnt3a-MSC group (15.0 \pm 0.28$)$ was significantly higher than that of the injury only (10.0 \pm 0.48$)$, MSC (12.57 \pm 0.48$)$, pLenti-MSC (12.42 \pm 0.48$)$, and Wnt3a-MSC (13.71 \pm 0.61 ) groups (Fig. 4).

\section{Immunofluorescence for axonal regeneration markers}

The expression of axonal regeneration markers was analyzed in a region proximal to the injury epicenter, where cells were injected. Confocal imaging revealed that the expression of axonal regeneration markers such as GAP43, MAP2, and NF were increased in the Wnt3a-MSC and 1.7 Wnt3a-MSC groups as compared with injury only, MSC, and pLenti-MSC groups (Figs. 5-7). However, it was difficult to find a significant difference between the Wnt3a-MSC and 1.7 Wnt3a-MSC groups (Figs. 5c, 6c, and 7c).
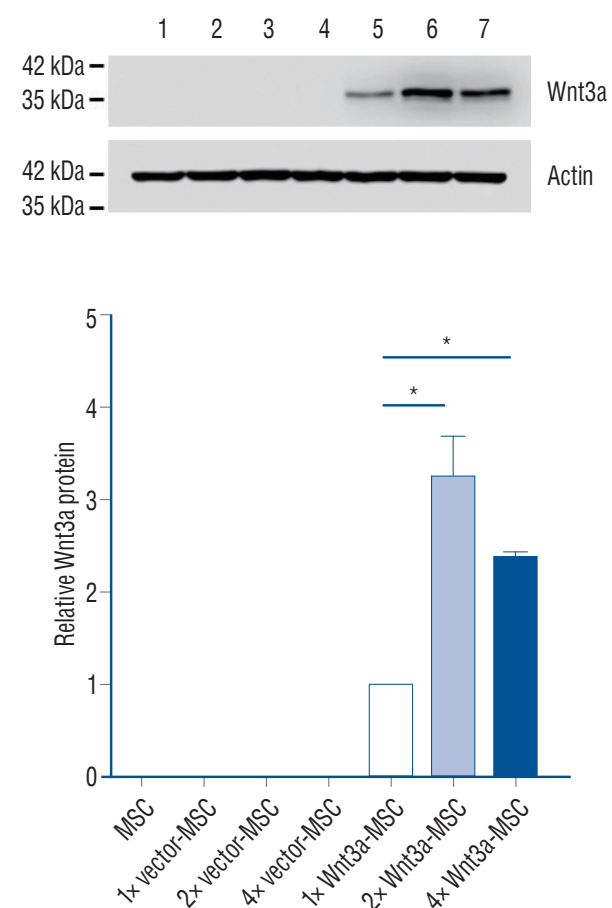

Fig. 2. Western blotting analysis of Wnt3a protein expression in each transfection condition. $2 \times$ Wnt3a-MSC showed 3.25 fold Wnt3a protein expression, and $4 \times$ Wnt3a-MSC showed 2.38 fold Wnt3a as compared to $1 \times$ Wnt3a-MSC. ${ }^{*} p<0.001$. MSC : mesenchymal stem cells.
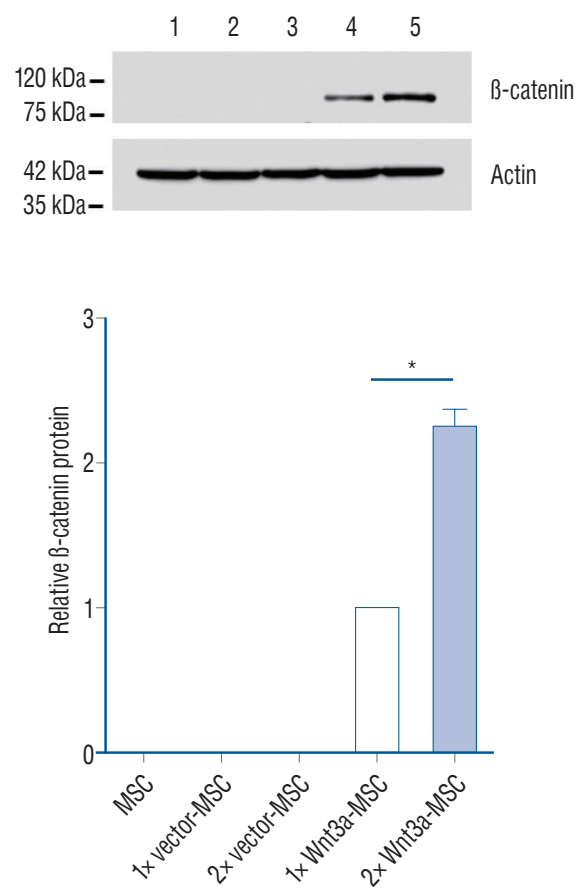

Fig. 3. Western blotting analysis. $\beta$-catenin expression in L929 cells indicates functionality of secreted Wnt3a protein. $2 \times$ Wnt3a-MSC showed a 2.2 fold increase in $\beta$-catenin expression level as compared to $1 \times$ Wnt3a-MSC. ${ }^{*} p<0.001$. MSC : mesenchymal stem cells.

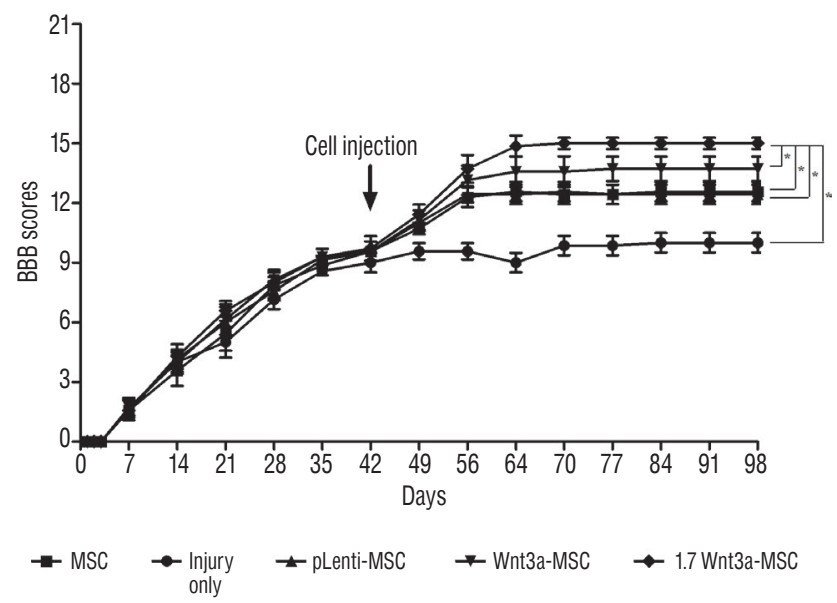

Fig. 4. Basso-Beattie-Bresnahan (BBB) locomotor scale test. We transplanted phosphate-buffered saline (injury only group), hMSC (MSC group), hMSC transfected with pLenti vector (pLenti-MSC group), hMSC transfected with Wnt3a gene (Wnt3a-MSC group), and hMSC transfected with enhanced Wnt3a gene (1.7 Wnt3a-MSC group) in each group of rat at 6 weeks after spinal cord injury (new chronic spinal cord injury model). The 1.7 Wnt3a-MSC group showed significant behavioral recovery as compared to all other groups at 8 weeks after transplantation. Data were presented as mean \pm standard deviation. ${ }^{*} p<0.05$. MSC : mesenchymal stem cells, hMSC : human mesenchymal stem cells. 


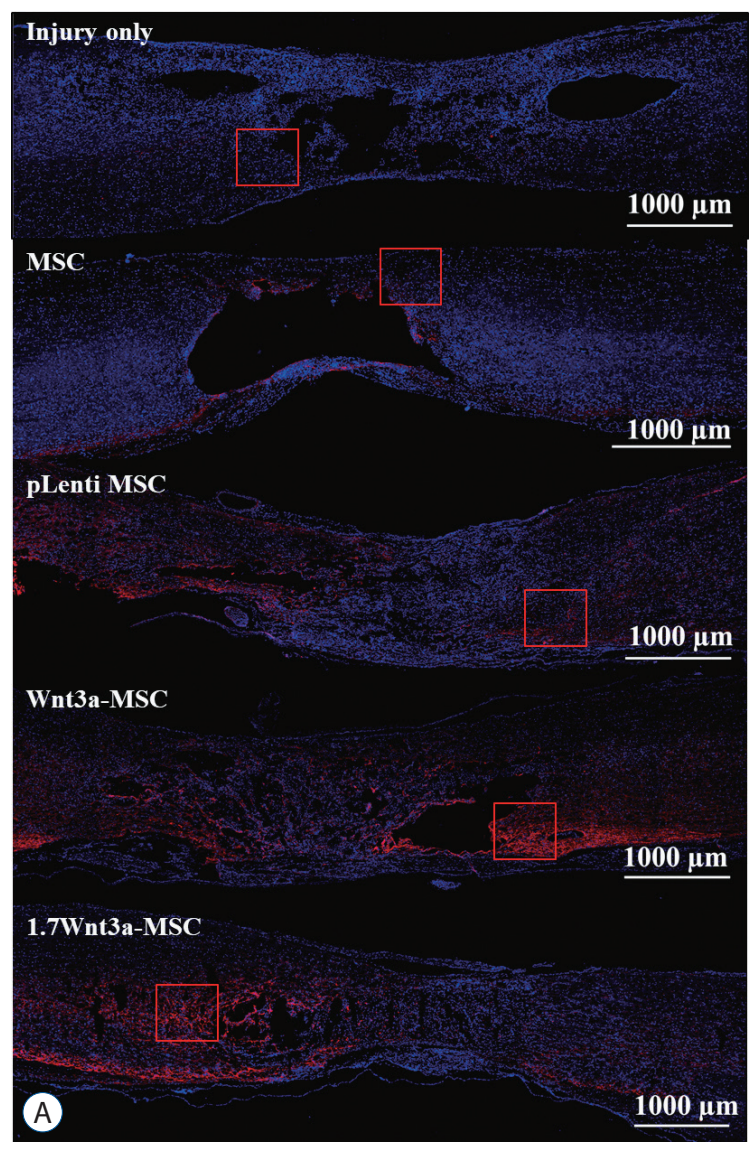

GAP43

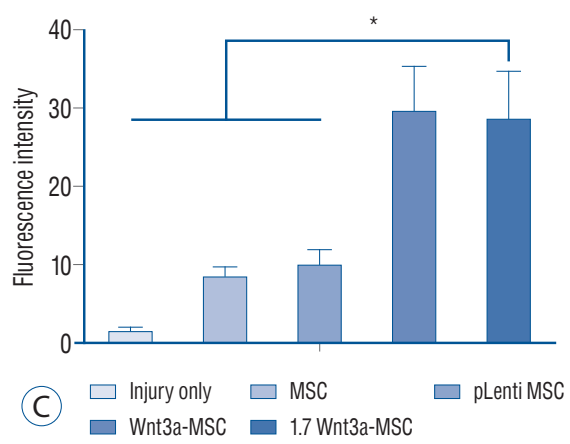

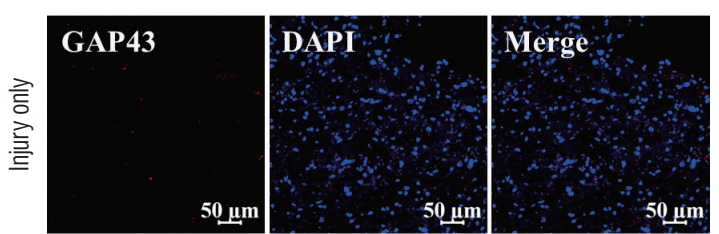
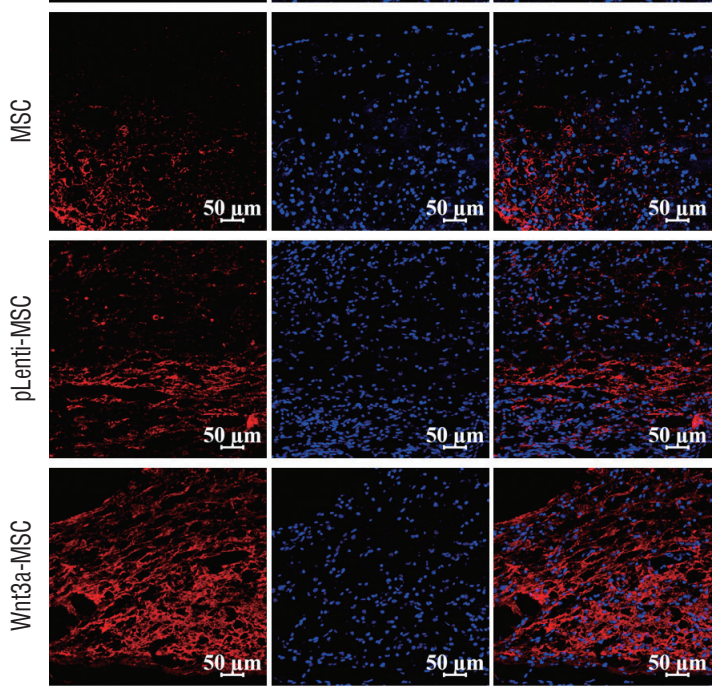

(B)

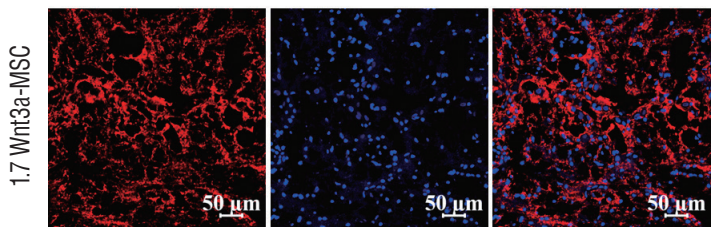

Fig. 5. Immunofluorescent staining of axonal regeneration marker growth-associated protein 43 (GAP43). Confocal microscopic images revealed that anti-GAP43 antibody staining in the spinal cord was greater in the Wnt3a-MSC and 1.7 Wnt3a-MSC groups than in other groups. A : Tile scan image; scale bar : $1000 \mu \mathrm{m}$. B : Scale bar : $50 \mu \mathrm{m}$ (magnification, $\times 100$ ). C : Quantification of fluorescence intensity. ${ }^{*} p<0.05$. MSC : mesenchymal stem cells, DAPI : 4',6-diamidino-2phenylindole.

\section{DISCUSSION}

We previously demonstrated the therapeutic effects of MSC treatment on neural regeneration via direct injection of cells into injured spinal cords of chronic SCI patients ${ }^{16,20)}$. However, in these two clinical trials, pure MSC did not show sufficient effects even though it demonstrated safety in the long-term follow-ups. Therefore, to maximize the neural regenerative effect through cell transplantation, we developed enhanced MSCs that secrete Wnt3a protein. There was also a need to calibrate the accuracy of the chronic SCI model in animals. We tested the efficacy of Wnt3a-secreting cell transplantations in regenerating injured axons in a rat model of SCI, and found a significant degree of axonal regeneration and recovery of motor func$\operatorname{tion}^{21,25,26)}$. However, there was a discrepancy between the clinical and animal studies. In the clinical studies, MSC were injected in chronic SCI patients. In the animal studies, we could not be certain whether the animal model used in the previous study was at the chronic or subacute stage of SCI. Although the time point used, i.e., 4 weeks after SCI, is generally 


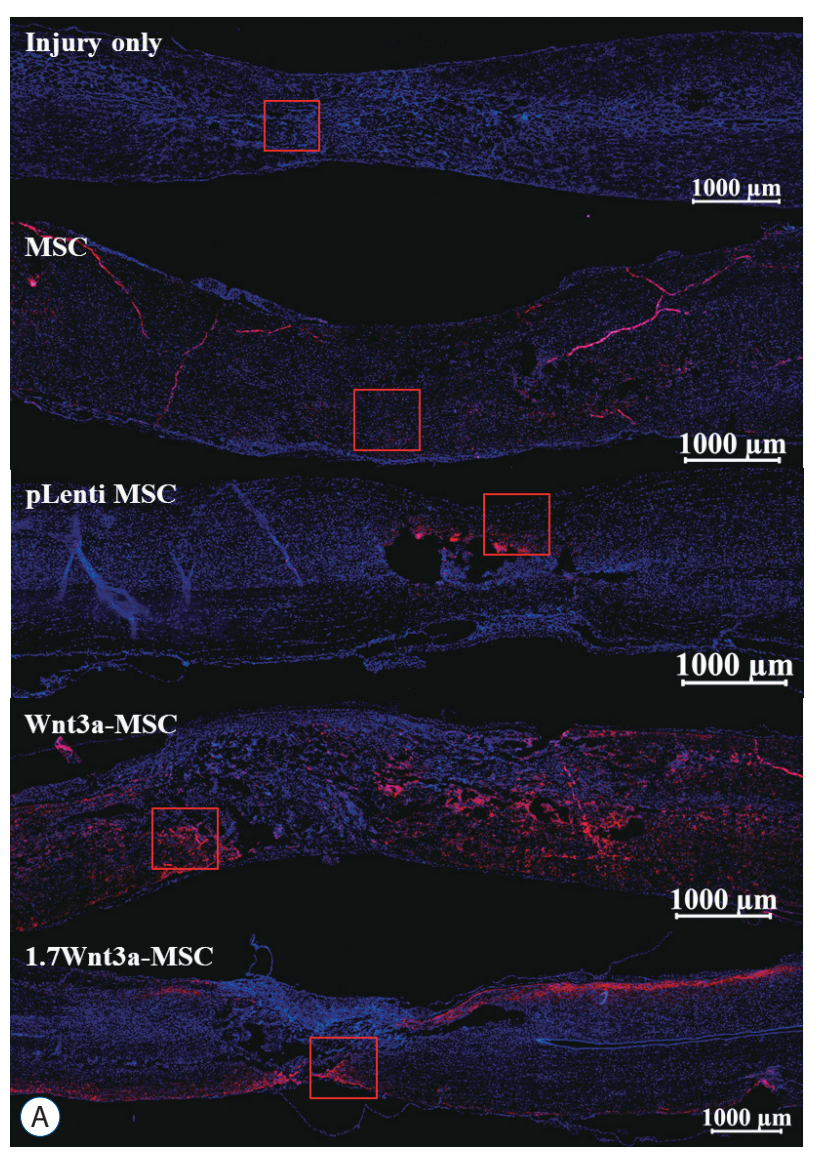

MAP2

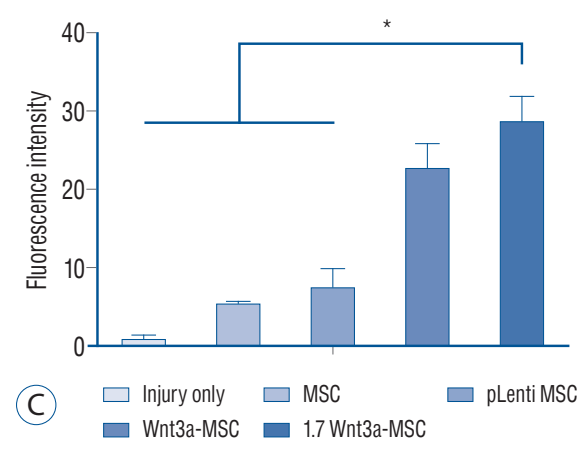

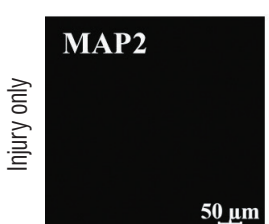
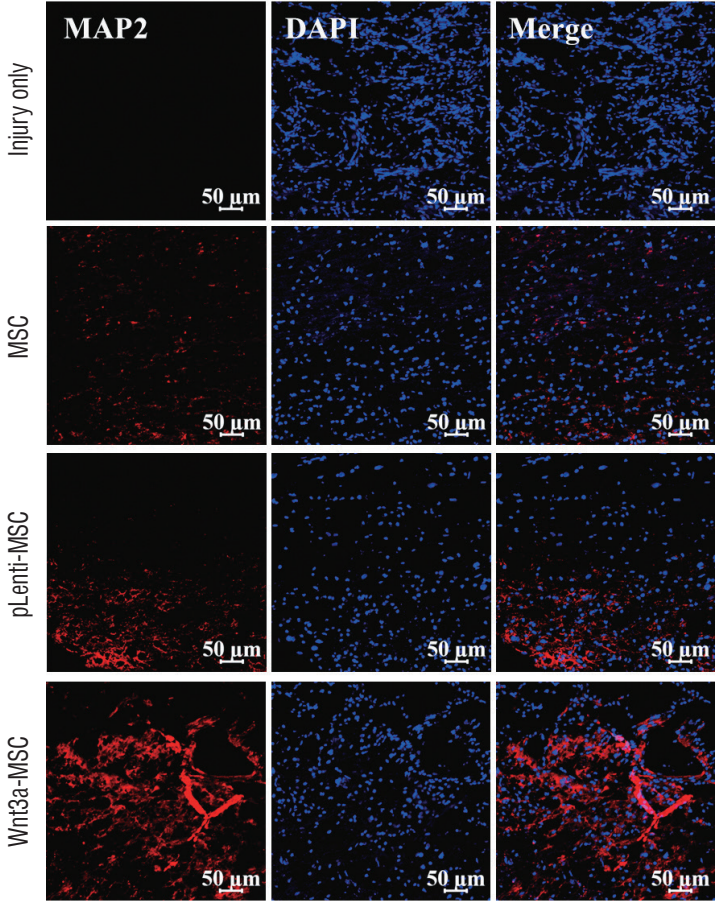

(B)
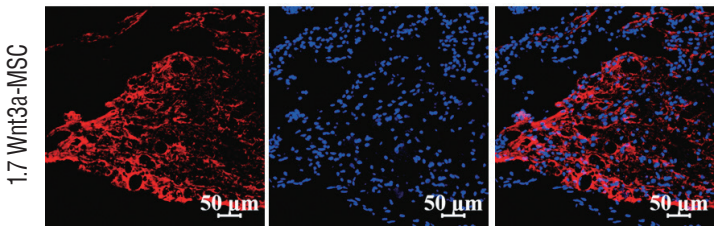

Fig. 6. Immunofluorescent staining of axonal regeneration marker microtubule-associated protein 2 (MAP2). Confocal microscopic images revealed that anti-MAP2 antibody staining in the spinal cord was greater in the Wnt3a-MSC and 1.7 Wnt3a-MSC groups than in other groups. A : Tile scan image; scale bar : $1000 \mu \mathrm{m}$. B : Scale bar : $50 \mu \mathrm{m}$ (magnification, $\times 100$ ). C : Quantification of fluorescence intensity. ${ }^{*} p<0.05$. MSC : mesenchymal stem cells, DAPI : 4',6-diamidino-2-phenylindole.

regarded as chronic SCI as per previous animal studies ${ }^{15)}$, we could observe that slight gradual BBB increase during 2 weeks even after 4 weeks of SCI. On the contrary, the clinical study performed with the definition of the chronic phase as after 12 months of injury with no neurological changes in the previous 6 months ${ }^{6,9)}$. Therefore, in this study, we decided upon the time point of 6 weeks after SCI in a rat model of chronic SCI, based on our previous pilot study (data not shown).

Several mechanisms behind the effectiveness of stem cell transplantation in recovering SCI have been proposed, such as differentiation of spinal cord neurons leading to tissue preservation, decreased injury size, and increased axonal regeneration $^{11,17,19,24)}$. Secreted trophic factors from MSC, such as nerve growth factor, vascular endothelial growth factor, and brainderived neurotrophic factor, are known to promote axonal regeneration and functional recovery as well ${ }^{4,24)}$. It is also possible that genetically engineered neural stem cells can exhibit better spinal cord repair and cell survival, and migrate longer distances $^{2,5,11}$. In the present study, we modified MSC with the Wnt3a gene to enhance the function of pure MSC. Whereas 


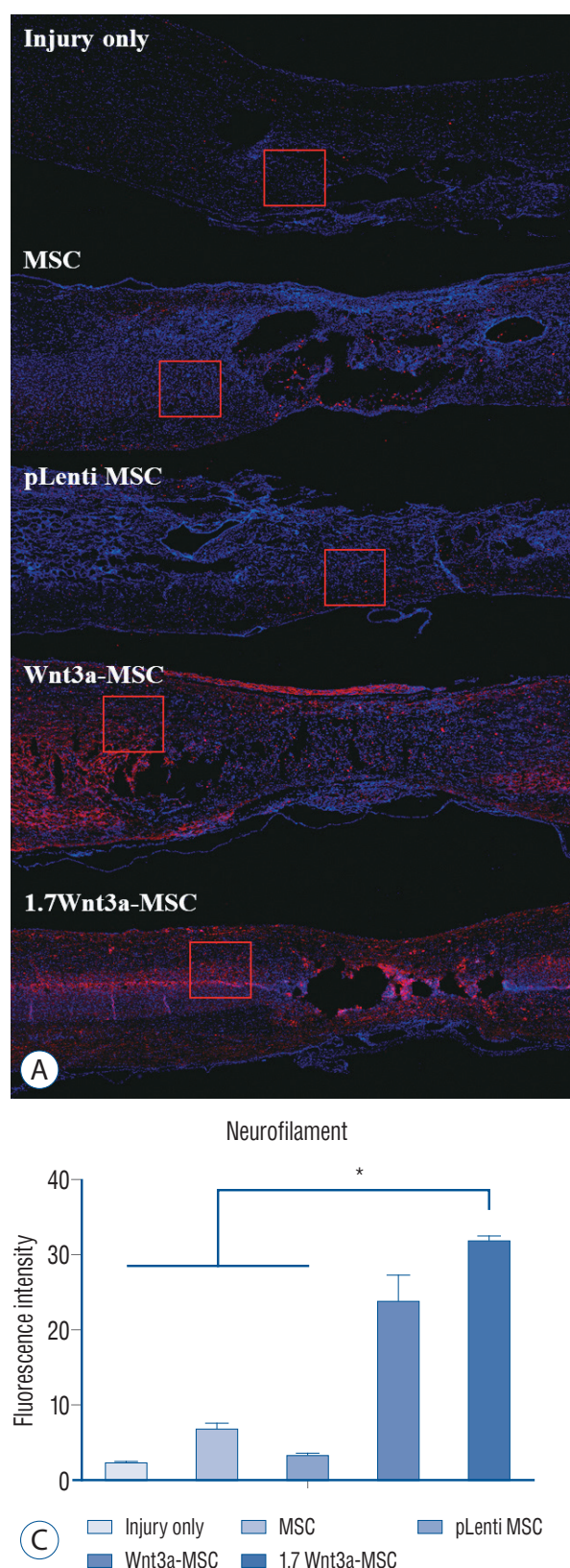

$1000 \mu \mathrm{m}$

$1000 \mu \mathrm{m}$

$1000 \mu \mathrm{m}$
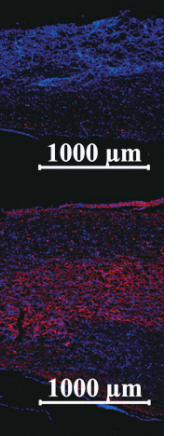
$1000 \mu \mathrm{m}$ (B)
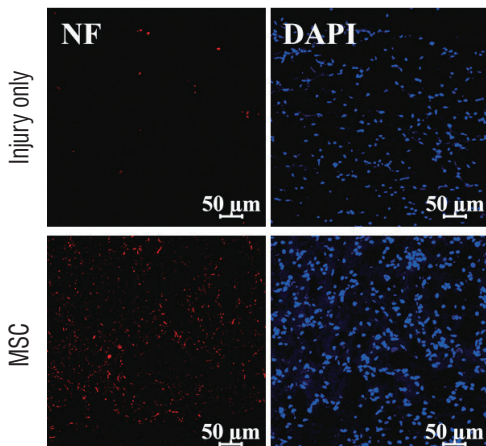

Merge
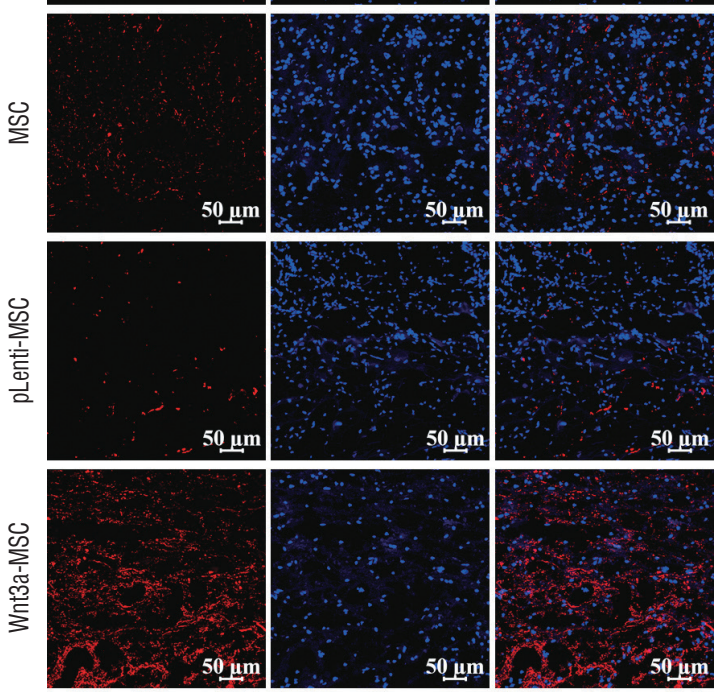

Fig. 7. Immunofluorescent staining of axonal regeneration marker neurofilament (NF). Confocal microscopic images revealed that anti-NF antibody staining in the spinal cord was greater in the Wnt3a-MSC and 1.7 Wnt3a-MSC groups than in other groups. A : Tile scan image; scale bar : 1000 $\mu \mathrm{m}$. B : Scale bar : $50 \mu \mathrm{m}$ (magnification, $\times 100$ ). C : Quantification of fluorescence intensity. ${ }^{*} p<0.05$. MSC : mesenchymal stem cells, DAPI : 4',6-diamidino-2-phenylindole. endogenous Wnt1 and Wnt5a signaling mediates only limited functional recovery after $\mathrm{SCI}^{7,13)}$, canonical Wnt3a signaling promotes spinal cord neural precursor development and stimulates axonal regeneration ${ }^{22,26)}$. We have also demonstrated that Wnt3a by itself, Wnt3a-secreting fibroblasts, and Wnt3asecreting fibroblasts with alginate scaffolds can improve functional recovery in SCI rat models ${ }^{21,26)}$. In this study, stem cell injection was performed using a stereotactic device and a microsyringe pump ( $1 \mu \mathrm{L} / \mathrm{min})$, and only a Hamilton syringe $(31 \mathrm{G})$ was inserted into the tissue, and there were no side ef- fects. In most of the animal studies, the injection method using a stereotactic device and a microsyringe pump is used, and in clinical studies, manual injection was also performed to the patient ${ }^{10,20)}$. In addition, regarding the rationales of the 2 point injection, we reported at the previous paper : the cavity usually developed in the injury epicenter, which has low tissue pressure. Therefore, when we inject the MSC in the injury epicenter, the cells are kept in the tissue due to low tissue pressure, but there is a disadvantage of low cell survival from lack of blood circulation. On the contrary, to inject cells at $2 \mathrm{~mm}$ 
Table 1. The ratio of detected cytokines in the conditioned media of each group

\begin{tabular}{lccc}
\hline Cytokine & $\mathbf{1 . 7} \mathbf{~ W n t 3 a / W n t 3 a}$ & $\mathbf{1 . 7}$ Wnt3a/MSC & Wnt3a/MSC \\
\hline BDNF & 1.2 & 1.4 & 1.2 \\
Beta-FGF & 1.3 & 1.2 & 0.9 \\
\hline Beta-NGF & 1.2 & 1.1 & 1.0 \\
EGF & 0.9 & 1.4 & 1.6 \\
FGF-4 & 1.0 & 1.3 & 1.2 \\
FGF-7 & 1.1 & 1.4 & 1.2 \\
GDNF & 0.9 & 1.4 & 1.4 \\
NT-3 & 1.2 & 1.2 & 1.0 \\
NT-4 & 1.1 & 1.3 & 1.2 \\
VEGF & 1.1 & 0.6 & 0.5 \\
\hline
\end{tabular}

MSC : mesenchymal stem cells, BDNF : brain-derived neurotrophic factor, FGF : fibroblast growth factors, EGF : epidermal growth factor, GDNF : glial cell line-derived neurotrophic factor, NT : neurotrophin, VEGF : vascular endothelial growth factor

proximal to epicenter has an advantage in cell survival, but there is a possibility of cell leakage ${ }^{20)}$.

We hypothesized that increasing the level of gene expression of Wnt3a in hMSC could highly promote axonal regeneration in injured spinal cords as compared to low-expressing Wnt3atransfected hMSC. Hence, we developed a new Wnt3a-MSC which was transfected with the Wnt3a gene $(2 \times, 4 \times)$. We selected the $2 \times$ Wnt3a-MSC as an enhanced Wnt3a-MSC, and referred to it as 1.7 Wnt3a-MSC because it showed 1.7 fold Wnt3a mRNA expression. The 1.7 Wnt3a-MSC showed higher $\beta$-catenin expression level when compared to baseline $(1 \times \mathrm{Wn}-$ t3a-MSC). A result of western blot of Wnt3a protein showed that $2 \times$ Wnt3a-MSC expressed more Wnt3a protein than $4 \times$ Wnt3a-MSC (Fig. 2). These data suggest that protein expression does not increase proportionally when mRNA is increased, and that the optimal volume of mRNA in the cell exists. It is known that the amount of mRNA and that of produced protein are not always proportional to each other, and the underlying mechanism in translation seems to be affected to the concentration of ribosomes, the effect of tRNA, and protein characteristics. In this study, we found out the optimal rate of Wnt3a mRNA is 1.7 Wnt3a-MSC ( $2 \times$ Wnt3a-MSC) ${ }^{12}$. We finally found that the $1.7 \mathrm{Wnt} 3 \mathrm{a}-\mathrm{MSC}$ showed the best titration result showing the best $\mathrm{BBB}$ improvement. In addition to Wnt3a, the cells of MSC, Wnt3a-MSC and 1.7 Wnt3a-MSC groups released different profiles of other neurotrophic factors (Table 1). The cells of Wnt3a group produced more secretions than the cell of MSC group in brain-derived neurotrophic factor (BDNF), epidermal growth factor (EGF), fibroblast growth factors (FGF)-4, FGF-7, glial cell line-derived neurotrophic factor
(GDNF), neurotrophin (NT)-4, and the cells of $1.7 \mathrm{Wnt} 3 \mathrm{a}$ group secreted more than the cells of Wnt3a group in BDNF, Beta-FGF, Beta-NGF, FGF-7, NT-3, NT-4, indicating that the three cytokines (BDNF, FGF-7, NT-4) especially showed linear increase in MSC, Wnt3a, and 1.7 Wnt3a groups, and they seemed to be persistently related to spinal cord regeneration (Table 1). It is well-known that BDNF, GDNF, NGF, and NT-3 are mainly effective neutrophic factors for spinal cord regeneration $^{3)}$. This information proved valuable when we applied the enhanced Wnt3a-MSC to SCI animal model. Therefore, we could name the 1.7 Wnt3a-MSC as a "smart stem cell" regarding functional recovery in SCI. However, since it is the result of one media analysis, statistical processing was not conducted, and statistical verification should be performed through multiple media analyses in the further study.

We performed $\mathrm{BBB}$ test to demonstrate functional recovery from SCI after injection of hMSC with enhanced Wnt3a gene expression. The 1.7 Wnt3a-MSC group showed significantly more improvement than other groups at 8 weeks after transplantation. Fourteen weeks after injury ( 8 weeks after transplantation), the scores of all groups plateaued. Thus, we selected this time point to be representative of final motor recovery and reasonable to assess locomotor improvement. In this study, we did not evaluate neuropathic pain, such as the von Frey test, but did only motor power recovery. In the further study, we are needed to investigate the neuropathic pain and bladder function recovery that are clinically common problems in chronic SCI. This will be a multiple aspect study for spinal cord regeneration.

Immunofluorescence was performed for visual analysis of 
axonal regeneration. GAP43, MAP2, and NF are well known representative markers of axonal regeneration. These markers were used to determine whether the increased behavioral test scores were the result of axonal regeneration or other neuroprotective effects. Because both the 1.7 Wnt3a-MSC and the Wnt3a-MSC groups showed higher expression of all axonal regeneration markers than the other groups, we can conclude that the BBB improvement was from axonal regeneration. However, we could not differentiate the differences in the staining of markers between the effects of 1.7 Wnt3a-MSC and Wnt3a-MSC injection in these rats in this study (Figs. 5-7). We speculate that enhanced Wnt3a gene MSC (1.7 Wnt3a-MSC) can exert stronger regeneration effect for injured spinal cord, and the result was evaluated by behavior test (BBB score), but unfortunately immunostaining with GAP43, MAP2, and NF showing spinal cord regeneration failed to demonstrate the precise difference of axon regeneration between Wnt3a-MSC and 1.7 Wnt3a-MSC group. To demonstrate the effect of spinal cord regeneration by Wnt3a gene enhancing with imaging technique, we need to additional imaging method such as axon tracing in further study. Basically, this study was investigated in the chronic phase of SCI which inflammation and tissue necrosis has already disappeared, and glial scar formation was set, in which neurological deficit only exists unlike phenomenon in acute or subacute phase. Therefore, analysis on the effect of angiogenesis, antiinflammation, and glial scar formation, which are important mechanisms of neuroprotection in acute or subacute phase of SCI, was not done to focus on the recovery mechanism for chronic status of SCI. In addition, we did not conduct the analysis of lesion cavity size or fibrosis change, which are another aspect to figure out the spinal cord regeneration. We consider that this is the limitation of our study for histology, and we are needed to add them in further study.

\section{CONCLUSION}

We produced 1.7 fold Wnt3a mRNA expressing hMSCs to promote the efficacy of axonal regeneration based on the safety of pure hMSCs and found that this gene titration is optimal to BBB improvement through axonal regeneration. Our study suggests that enhanced gene expression with optimal ratio in gene transfected MSC can significantly impact axonal regen- eration and functional recovery in a rat model of chronic SCI more than pure hMSC.

\section{CONFLICTS OF INTEREST}

No potential conflict of interest relevant to this article was reported.

\section{INFORMED CONSENT}

This type of study does not require informed consent.

\section{AUTHOR CONTRIBUTIONS}

\author{
Conceptualization : SRJ, SWK, HL \\ Data curation : JM, HJL \\ Formal analysis : JM, JHK \\ Funding acquisition : SRJ, JHK \\ Methodology : SWK, SRJ \\ Project administration : SRJ \\ Visualization : HHY, JM \\ Writing - original draft : HHY, JHP \\ Writing - review \& editing : SRJ, JHK
}

\section{ORCID}

$\begin{array}{ll}\text { Hyung Ho Yoon } & \text { https://orcid.org/0000-0003-4123-0112 } \\ \text { Hyang Ju Lee } & \text { https://orcid.org/0000-0001-5558-5550 } \\ \text { Joongkee Min } & \text { https://orcid.org/0000-0002-1680-4797 } \\ \text { Jeong Hoon Kim } & \text { https://orcid.org/0000-0001-7131-4523 } \\ \text { Jin Hoon Park } & \text { https://orcid.org/0000-0002-0903-3146 } \\ \text { Ji Hyun Kim } & \text { https://orcid.org/0000-0002-1738-1597 } \\ \text { Seong Who Kim } & \text { https://orcid.org/0000-0002-3731-9109 } \\ \text { Heuiran Lee } & \text { https://orcid.org/0000-0002-4241-5664 } \\ \text { Sang Ryong Jeon } & \text { https://orcid.org/0000-0002-8340-7978 }\end{array}$

\section{- Acknowledgements}

This research was supported by a grant of the Korea Health Technology R\&D Project through the Korea Health Industry 
Development Institute (KHIDI), funded by the Ministry of Health \& Welfare, Republic of Korea (grant number : HI16C2188), and funded by a grant (18-176 and 19-176) from the Asan Institute for Life Science, Seoul, Korea.

\section{References}

1. Basso DM, Beattie MS, Bresnahan JC : A sensitive and reliable locomotor rating scale for open field testing in rats. J Neurotrauma $12: 1-21$, 1995

2. Blits B, Kitay BM, Farahvar A, Caperton CV, Dietrich WD, Bunge MB : Lentiviral vector-mediated transduction of neural progenitor cells before implantation into injured spinal cord and brain to detect their migration, deliver neurotrophic factors and repair tissue. Restor Neurol Neurosci $23: 313-324,2005$

3. Boyce VS, Mendell LM : Neurotrophic factors in spinal cord injury. Handb Exp Pharmacol 220 : 443-460, 2014

4. Chao MV : Neurotrophins and their receptors: a convergence point for many signalling pathways. Nat Rev Neurosci 4 : 299-309, 2003

5. Dinsmore J, Ratliff J, Deacon T, Pakzaban P, Jacoby D, Galpern W, et al. : Embryonic stem cells differentiated in vitro as a novel source of cells for transplantation. Cell Transplant 5 : 131-143 1996

6. Fawcett JW, Curt A, Steeves JD, Coleman WP, Tuszynski MH, Lammertse $D$, et al. : Guidelines for the conduct of clinical trials for spinal cord injury as developed by the ICCP panel: spontaneous recovery after spinal cord injury and statistical power needed for therapeutic clinical trials. Spinal Cord 45 : 190-205, 2007

7. Hirabayashi $Y$, Itoh $Y$, Tabata H, Nakajima K, Akiyama T, Masuyama N, et al. : The Wnt/beta-catenin pathway directs neuronal differentiation of cortical neural precursor cells. Development $131: 2791-2801,2004$

8. Huelsken J, Behrens J : The Wnt signalling pathway. J Cell Sci 115 : 3977-3978, 2002

9. Jeong SK, Choi I, Jeon SR : Current status and future strategies to treat spinal cord injury with adult stem cells. J Korean Neurosurg Soc 63 : 153-162, 2020

10. Kutikov AB, Moore SW, Layer RT, Podell PE, Sridhar N, Santamaria AJ, ed al. : Method and apparatus for the automated delivery of continuous neural stem cell trails into the spinal cord of small and large animals. Neurosurgery 85 : 560-573, 2019

11. McDonald JW, Liu XZ, Qu Y, Liu S, Mickey SK, Turetsky D, et al. : Transplanted embryonic stem cells survive, differentiate and promote recovery in injured rat spinal cord. Nat Med 5 : 1410-1412, 1999

12. Mehra $A$, Lee $K H$, Hatzimanikatis $V$ : Insights into the relation between mRNA and protein expression patterns: I. Theoretical considerations. Biotechnol Bioeng 84 : 822-833, 2003

13. Miyashita T, Koda M, Kitajo K, Yamazaki M, Takahashi K, Kikuchi A, et al. : Wnt-Ryk signaling mediates axon growth inhibition and limits functional recovery after spinal cord injury. J Neurotrauma 26 : 955-964, 2009
14. Mukhamedshina YO, Akhmetzyanova ER, Kostennikov AA, Zakirova EY, Galieva LR, Garanina EE, et al. : Adipose-derived mesenchymal stem cell application combined with fibrin matrix promotes structural and functional recovery following spinal cord injury in rats. Front Pharmacol 9 : 343, 2018

15. Nakano N, Nakai Y, Seo TB, Homma T, Yamada Y, Ohta M, et al. : Effects of bone marrow stromal cell transplantation through CSF on the subacute and chronic spinal cord injury in rats. PLoS One 8 : e73494, 2013

16. Oh SK, Choi KH, Yoo JY, Kim DY, Kim SJ, Jeon SR : A phase III clinical trial showing limited efficacy of autologous mesenchymal stem cell therapy for spinal cord injury. Neurosurgery 78 : 436-447; discussion 447, 2016

17. Ohta M, Suzuki Y, Noda T, Ejiri Y, Dezawa M, Kataoka K, et al. : Bone marrow stromal cells infused into the cerebrospinal fluid promote functional recovery of the injured rat spinal cord with reduced cavity formation. Exp Neurol 187 : 266-278, 2004

18. Onishi $K$, Hollis E, Zou $Y$ : Axon guidance and injury-lessons from Wnts and Wnt signaling. Curr Opin Neurobiol : 232-240, 2014

19. Osaka M, Honmou O, Murakami T, Nonaka T, Houkin K, Hamada H, et al. : Intravenous administration of mesenchymal stem cells derived from bone marrow after contusive spinal cord injury improves functional outcome. Brain Res 1343 : 226-235, 2010

20. Park JH, Kim DY, Sung IY, Choi GH, Jeon MH, Kim KK, et al. : Longterm results of spinal cord injury therapy using mesenchymal stem cells derived from bone marrow in humans. Neurosurgery 70 : 1238-1247; discussion 1247, 2012

21. Park JH, Min J, Baek SR, Kim SW, Kwon IK, Jeon SR : Enhanced neuroregenerative effects by scaffold for the treatment of a rat spinal cord injury with Wnt3a-secreting fibroblasts. Acta Neurochir (Wien) 155 : 809-816, 2013

22. Parr BA, Shea MJ, Vassileva G, McMahon AP : Mouse Wnt genes exhibit discrete domains of expression in the early embryonic CNS and limb buds. Development 119 : 247-261, 1993

23. Patapoutian A, Reichardt LF : Roles of Wnt proteins in neural development and maintenance. Curr Opin Neurobiol 10 : 392-399, 2000

24. Samdani AF, Paul C, Betz RR, Fischer I, Neuhuber B : Transplantation of human marrow stromal cells and mono-nuclear bone marrow cells into the injured spinal cord: a comparative study. Spine (Phila Pa 1976) 34 : 2605-2612, 2009

25. Seo DK, Kim JH, Min J, Yoon HH, Shin ES, Kim SW, et al. : Enhanced axonal regeneration by transplanted Wnt3a-secreting human mesenchymal stem cells in a rat model of spinal cord injury. Acta Neurochir (Wien) 159 : 947-957, 2017

26. Suh HI, Min J, Choi KH, Kim SW, Kim KS, Jeon SR : Axonal regeneration effects of Wnt3a-secreting fibroblast transplantation in spinal cordinjured rats. Acta Neurochir (Wien) 153 : 1003-1010, 2011

27. Verma $R$, Virdi JK, Singh $N$, Jaggi AS : Animals models of spinal cord contusion injury. Korean J Pain 32 : 12-21, 2019

28. Zou Y : Wnt signaling in axon guidance. Trends Neurosci $27: 528$ 532,2004 